\title{
On a problem of Marco Buratti
}

\author{
Peter Horak \\ University of Washington \\ Tacoma, WA 98402 \\ horak@u . washington.edu
}

\author{
Alexander Rosa \\ McMaster University \\ Hamilton, Ontario, Canada \\ rosa@mcmaster.ca
}

Submitted: Apr 9, 2008; Accepted: Jan 21, 2009; Published: Feb 4, 2009

Mathematics Subject Classification: 05C38

\begin{abstract}
We consider a problem formulated by Marco Buratti concerning Hamiltonian paths in the complete graph on $Z_{p}, p$ an odd prime.
\end{abstract}

\section{Introduction}

Let $K_{p}$ be the complete graph on $p$ vertices. We will usually take $Z_{p}$, the cyclic group of order $\mathrm{p}$, as the set of vertices of $K_{p}$. The length of the edge $u v, u, v \in K_{p}$ (or the distance of $u$ and $v)$ is given by $d(u, v)=\min (|u-v|, p-|u-v|)$. Given a path $P=\left(v_{1}, v_{2}, \ldots, v_{m}\right)$, we denote the multiset of edge-lengths of $P$ by $d(P): d(P)=\left\{d\left(v_{i}, v_{i+1}\right): i=1,2, \ldots, m-1\right\}$.

Marco Buratti [1] formulated the following problem:

Let $p=2 n+1$ be a prime, let $L$ be any list of $2 n$ elements, each from the set $\{1,2, \ldots, n\}$. Does there exist a Hamiltonian path $H$ in $K_{p}$ with $V\left(K_{p}\right)=Z_{p}$ such that the set of edge-lengths of $H$ comprises $L$ ? (That is, such that $d(H)=L$ ?)

He conjectured that the answer is yes for every list $L$.

A realization of a list $L$ is a Hamiltonian path $\left(x_{0}, x_{1}, \ldots, x_{2 n}\right)$ on vertices of $Z_{p}$ such that the (multi)-set of edge-lengths $\left\{d\left(x_{i}, x_{i+1}\right): 0,1, \ldots, 2 n-1\right\}$ equals $L$. In other words, Buratti's conjecture says that every such list $L$ has a realization (or is realizable).

If a list $L$ consists of $a_{1} 1$ 's, $a_{2} 2$ 's, $\ldots, a_{n} n$ 's, where $a_{1}+a_{2}+\ldots a_{n}=2 n$, we will use exponential notation for $L$ and write $L=1^{a_{1}} 2^{a_{2}} \ldots n^{a_{n}}$ or, alternatively, we will say that $L$ is of type $\left[a_{1}, a_{2}, \ldots, a_{n}\right]$, or for the sake of brevity, we will write simply $L=\left[a_{1}, a_{2}, \ldots, a_{n}\right]$.

To best of our knowledge, no results on Buratti's conjecture have been published so far. However, using a computer, Mariusz Meszka [3] has verified the validity of Buratti's conjecture for all primes $p \leq 23$.

The problem does not appear to be easy. The purpose of this article is to present some initial ideas, approaches and results towards the complete solution of Buratti's conjecture.

One such approach is outlined in Section 2 where certain graphs having lists as vertices, arranged in lexicographic order, are considered. Some properties of the smallest list 
without a realization, if such exists, are derived. In Section 3 we prove that certain classes of lists are realizable. In particular, we show that any list where one of the distances occurs "sufficiently many times" is realizable. We also show that any list consisting of just two distinct distances is realizable.

The general case of lists with only two distances, that is, when $p$ is any positive integer, not just a prime, is characterized in Section 4. This characterization is a clear indication of the complexity of the problem.

\section{Graphs on lists}

Let $p=2 n+1$ be a prime. In what follows we consider lists of $2 n$ elements, each from $\{1,2, \ldots, n\}$.

Let $\mathcal{L}_{p}$ be the set of such lists. Clearly, we have $\left|\mathcal{L}_{p}\right|=\left(\begin{array}{c}3 n-1 \\ n-1\end{array}\right)$. Define the graph $\mathcal{G}_{p}$ as follows: $V\left(\mathcal{G}_{p}\right)=\mathcal{L}_{p}$. For two lists $L, L^{\prime} \in \mathcal{L}_{p}, L=\left[a_{1}, a_{2}, \ldots, a_{n}\right], L^{\prime}=\left[a_{1}{ }^{\prime}, a_{2}{ }^{\prime}, \ldots, a_{n}{ }^{\prime}\right]$, $\left\{L, L^{\prime}\right\} \in E\left(\mathcal{G}_{p}\right)$ when $\delta\left(L, L^{\prime}\right)=1$. Here $\delta\left(L, L^{\prime}\right)=\frac{1}{2} \sum_{i=1}^{n}\left|a_{i}-a_{i}{ }^{\prime}\right|$ is the distance between the two lists $L, L^{\prime}$ (which coincides with the distance in the graph $\mathcal{G}_{p}$ ). In other words, $L$ and $L^{\prime}$ are adjacent in $\mathcal{G}_{p}$ precisely when increasing one of the $a_{i}$ s in $L$ by one while decreasing another by one results in $L^{\prime}$.

We may view any realization of $L$ as a permutation $x_{0} x_{1} x_{2} \ldots x_{2 n}$ of $\{0,1, \ldots, 2 n\}$. Thus there is a totality of $(2 n+1)$ ! realizations of various lists $L$ (possibly these are not all the lists $L \in \mathcal{L}_{p}$ ).

Given a Hamiltonian path $\left(x_{0}, x_{1}, \ldots, x_{2 n}\right)$, we may delete the edge $x_{i} x_{i+1}$, for $i \in$ $\{0,1, \ldots, 2 n-1\}$, and replace it with either the edge $x_{0} x_{i+1}$ or with the edge $x_{i} x_{2 n}$ or with the edge $x_{0} x_{2 n}$, obtaining in each case another Hamiltonian path. Any such replacement will be called an $\alpha$-transformation. Notice that for $i=0$ or $i=2 n-1$ the three possibilities reduce to a single one, thus applying $\alpha$-transformations to any realization $L$ yields altogether $6 n-4$ realizations of (not necessarily distinct) lists.

Furthermore, replacing the Hamiltonian path $\left(x_{0}, x_{1}, \ldots, x_{i}, x_{i+1}, \ldots, x_{2 n}\right)$ with the Hamiltonian path $\left(x_{0}, x_{1}, \ldots, x_{i+1}, x_{i}, \ldots, x_{2 n}\right)$ for $i \in\{0,1, \ldots, 2 n-1\}$, that is, performing an adjacent transposition, will be called a $\beta$-transformation.

Define now the graphs $\mathcal{A}_{p}$ and $\mathcal{B}_{p}$ as follows:

The vertices of both, $\mathcal{A}_{p}$ and $\mathcal{B}_{p}$ are all $(2 n+1)$ ! realizations of lists $L=\left[a_{1}, a_{2}, \ldots, a_{2 n}\right]$. Two vertices are adjacent in $\mathcal{A}_{p}$ if one can be obtained from the other by an $\alpha$-transformation, and are adjacent in $\mathcal{B}_{p}$ if one can be obtained from the other by a $\beta$-transformation.

Our first observation follows from a well-known result on generating permutations by adjacent transpositions (see, e.g., [2]).

Lemma 2.1 The graph $\mathcal{B}_{p}$ is connected.

In fact, $\mathcal{B}_{p}$ is Hamiltonian. Similar statements hold for the graph $\mathcal{A}_{p}$.

Lemma 2.2 The graph $\mathcal{A}_{p}$ is connected. 
Proof. We need to show that for any two vertices $P, P^{\prime}$ of $\mathcal{A}_{p}$ there is a path in $\mathcal{A}_{p}$ from $P$ to $P^{\prime}$. Since the graph $\mathcal{B}_{p}$ is connected, there is a path in $\mathcal{B}_{p}$ from $P$ to $P^{\prime}$, say, $P, P_{1}, P_{2}, \ldots, P_{s}, P^{\prime}$. That is, $P$ and $P_{1}$ are adjacent in $\mathcal{B}_{p}$ which means that $P_{1}$ is obtained from $P$ by a transposition. Let $P=\left(x_{1}, x_{2}, \ldots, x_{2 n}\right)$. If the transposition is $\left(x_{1}, x_{2}\right)$ or $\left(x_{2 n-1}, x_{2 n}\right)$ then $P_{1}$ may be viewed as obtained from $P$ by an $\alpha$-transformation, i.e. by replacing the edge $\left(x_{2}, x_{3}\right)$ with $\left(x_{1}, x_{3}\right)$ (and similarly, if the transposition is $\left(x_{2 n-1}, x_{2 n}\right)$ ). So assume w.l.o.g that

$$
\begin{aligned}
& P=\left(x_{1}, x_{2}, \ldots, x_{s-1}, x_{s}, x_{s+1}, x_{s+2}, \ldots, x_{2 n-1}, x_{2 n}\right), \\
& P_{1}=\left(x_{1}, x_{2}, \ldots, x_{s-1}, x_{s+1}, x_{s}, x_{s+2}, \ldots, x_{2 n-1}, x_{2 n}\right)
\end{aligned}
$$

where $s \in\{2,3, \ldots, 2 n-2\}$ and the transposition is $\left(x_{s}, x_{s+1}\right)$. Perform consecutively the following $\alpha$-transformations: In $P$, replace the edge $\left\{x_{s-1} x_{s}\right\}$ with the edge $\left\{x_{s-1} x_{2 n}\right\}$ to obtain the path

$$
M_{1}=\left(x_{1}, x_{2}, \ldots, x_{s-1} x_{2 n} x_{2 n-1}, \ldots, x_{s+2}, x_{s+1}, x_{s}\right) .
$$

In $M_{1}$, replace the edge $\left\{x_{s+1}, x_{s+2}\right\}$ with the edge $\left\{x_{s}, x_{s+2}\right\}$ to obtain the path $M_{2}=\left(x_{1}, x_{2}, \ldots, x_{s-1}, x_{2 n}, x_{2 n-1}, \ldots, x_{s+2}, x_{s}, x_{s+1}\right)$.

In $M_{2}$, replace the edge $\left\{x_{s-1}, x_{2 n}\right\}$ with the edge $\left\{x_{s-1}, x_{s+1}\right\}$ to obtain the path $P_{1}=\left(x_{1}, x_{2}, \ldots, x_{s-1}, x_{s+1}, x_{s}, x_{s+2}, \ldots, x_{2 n-1}, x_{2 n}\right)$.

A similar sequence of $\alpha$-transformations will transform $P_{1}$ into $P_{2}, P_{2}$ into $P_{3}, \ldots, P_{s}$ into $P^{\prime}$, thus there is a path in $\mathcal{A}_{p}$ from $P$ to $P^{\prime}$.

The graph $\mathcal{A}_{p}{ }^{*}$, the reduced graph of $\mathcal{A}_{p}$ [the graph $\mathcal{B}_{p}{ }^{*}$, the reduced graph of $\mathcal{B}_{p}$, respectively], has as its vertex set the set $\mathcal{L}_{p}$ and is obtained by "contracting" in $\mathcal{A}_{p}$ [in $\mathcal{B}_{p}$, respectively] to a single vertex all realizations of a given list $L \in \mathcal{L}_{p}$ while suppressing all loops and multiple edges. Clearly, the graph $\mathcal{A}_{p}{ }^{*}$ is a subgraph of $\mathcal{G}_{p}$, and both $\mathcal{A}_{p}{ }^{*}$ and $\mathcal{B}_{p}{ }^{*}$ are connected.

Given a list $L=\left[a_{1}, a_{2}, \ldots, a_{n}\right]$, we may assume w.l.o.g. that $a_{1} \geq a_{i}$ for $i=2,3, \ldots, n$ since $p=2 n+1$ is a prime. (Namely, if $a_{k} \geq a_{i}$ instead for some $k$ and all $i \neq k$, replace each $i$ with $x . i$ where $x$ is such that $k . x=1$.) Let $\mathcal{G}_{p}{ }^{*}\left(\mathcal{A}_{p}{ }^{* *}\right.$ and $\mathcal{B}_{p}{ }^{* *}$, respectively) be the subgraph of $\mathcal{G}_{p}$ (the subgraph of $\mathcal{A}_{p}{ }^{*}$, and of $\mathcal{B}_{p}{ }^{*}$, respectively), induced by all vertices $L$ of $\mathcal{G}_{p}$ (of $\mathcal{A}_{p}{ }^{*}$, and of $\mathcal{B}_{p}{ }^{*}$, respectively) for which $a_{1} \geq a_{i}, i=2,3, \ldots, n$. Thus the largest vertex of $\mathcal{G}_{p}{ }^{*}$ in the lexicographic ordering of its elements is $\bar{L}=[2,2, \ldots, 2]$.

Lemma 2.3 The following are equivalent:

1. Buratti's conjecture is true.

2. The graph $\mathcal{A}_{p}{ }^{*}$ is a spanning subgraph of $\mathcal{G}_{p}$.

3. The graph $\mathcal{B}_{p}{ }^{*}$ is a spanning subgraph of $K_{\left|\mathcal{L}_{p}\right|}$ where $V\left(K_{\left|\mathcal{L}_{p}\right|}\right)=\mathcal{L}_{p}$.

4. The graph $\mathcal{A}_{p}{ }^{* *}$ is a spanning subgraph of $\mathcal{G}_{p}{ }^{*}$.

5. The graph $\mathcal{B}_{p}{ }^{* *}$ is a spanning subgraph of $\mathcal{G}_{p}{ }^{*}$. 
So far we are unable to prove any of 2., 3., 4., or 5. above. However, we are able to say something about the list $L^{*}$, the lexicographically smallest (in $\mathcal{G}_{p}$ or in $\mathcal{G}_{p}{ }^{*}$ ) list for which there does not exist any realization.

First we prove some lemmas.

Lemma 2.4 Let $L \in V\left(\mathcal{G}_{p}{ }^{*}\right)$ be a vertex adjacent to $\bar{L}=[2,2, \ldots, 2]$. Then $L$ has a realization.

Proof. Given $p=2 n+1$, one realization of $\bar{L}=[2,2, \ldots, 2]$ is $(0,1,2 n, 2,2 n-$ $1,3,2 n-2, \ldots, n+2, n, n+1)$. One possible $\alpha$-transformation that one may perform consists in replacing one edge of length $j \in\{1,2, \ldots, n-1\}$ with the edge $\{0, n+1\}$ of length $n$, resulting in a realization of the list $L=[2,2, \ldots, 2,1,2, \ldots, 2,3]$, that is, $a_{j}=1$ for some $j \in\{1,2, \ldots, n-1\}, a_{n}=3$, and $a_{i}=2$ for all other $i \neq j$. Let now $x$ be a primitive root of $G F(p)$, and let $s$ be such that $x^{s} n=1$. Then since $\left\{ \pm x^{s} j: j=\right.$ $1,2, \ldots, n-1\}=\{2,3, \ldots, n\}$, it follows that the set of lists $\left\{\left[a_{1}, a_{2}, \ldots, a_{j}, \ldots, a_{n}\right]: a_{1}=\right.$ $3, a_{j}=1, a_{i}=2$ for $\left.i=2,3, \ldots, n-1, i \neq j\right\}, j=2,3, \ldots, n-1$ is realizable if the set of lists $\left\{\left[a_{1}, a_{2}, \ldots, a_{j}, \ldots, a_{n}\right]: a_{j}=1, a_{n}=3, a_{i}=2\right.$ for $\left.i=1,2, \ldots, n-1, i \neq j\right\}$, $j=1,2, \ldots, n-1$ is realizable. This completes the proof.

On the set $\mathcal{L}_{p}$, let $<$ be the usual lexicographic order.

Lemma 2.5 Let $L^{*}=\left[a_{1}{ }^{*}, a_{2}{ }^{*}, \ldots, a_{n}{ }^{*}\right]$ be the lexicographically smallest list in $\mathcal{L}_{p}$ which has no realization. Let $\mathcal{L}_{p}{ }^{\prime}=\left\{L:\left\{L, L^{*}\right\} \in E\left(\mathcal{G}_{p}\right), L<L^{*}\right\}$. Then, for a given $L \in \mathcal{L}_{p}{ }^{\prime}$, $L=\left[b_{1}, b_{2}, \ldots, b_{n}\right]$, we have:

(i) there are $b_{r}, b_{s}, r<s$ such that $b_{s}-a_{s}{ }^{*}=a_{r}{ }^{*}-b_{r}=1$ (and $b_{i}=a_{i}{ }^{*}$ for $\left.i \neq r, s\right)$;

(ii) in any realization of $L$, say, $\left(0, x_{1}, \ldots, x_{2 n-1}, x_{2 n}\right), x_{2 n} \neq s, 2 n+1-s$;

(iii) if $\delta\left(x_{i}, x_{i+1}\right)=r$ and $x_{i+1}-x_{i}=r$ then $x_{i+1} \neq s, 2 n+1-s$, but if $x_{i}-x_{i+1}=r$ then $x_{i}-x_{2 n+1} \neq s, 2 n+1-s$.

Proof. (i) follows from the definition of the graph $\mathcal{G}_{p}$ and of $\mathcal{L}_{p}{ }^{\prime}$. (ii) If in a realization $\left(0, x_{1}, \ldots, x_{2 n}\right)$ of $L \in \mathcal{L}_{p}{ }^{\prime}, x_{2 n}=s$ or $x_{2 n}=2 n+1-s$ then an $\alpha$-transformation consisting in deleting the edge $\left\{x_{i}, x_{i+1}\right\}$ with $\delta\left(x_{i}, x_{i+1}\right)=s$ and replacing it with the edge $\{0, s\}$ results in a realization of $L^{*}$, a contradiction. (iii) If $x_{i+1}-x_{i}=r$ and $x_{i+1}=s$ then an $\alpha-$ transformation replacing the edge $\left\{x_{i}, x_{i+1}\right\}$ with the edge $\left\{0, x_{i+1}\right\}$ results in a realization of $L^{*}$, a contradiction, and similarly for the remaining cases.

Theorem 2.6 Let $L^{*}=\left[a_{1}{ }^{*}, a_{2}{ }^{*}, \ldots, a_{2 n}{ }^{*}\right]$ be the lexicographically smallest list in $\mathcal{L}_{p}{ }^{*}$ which does not have any realization. Then $3 \leq a_{1}{ }^{*} \leq 2 n-5$.

Proof. The right inequality follows from Corollary 3.3 (see Section 3 below) and the left one from Lemma 2.4 . 


\section{Realizable lists}

In this section, $L=\left\{d_{1}^{a_{1}}, \ldots, d_{k}^{a_{k}}\right\}$ will stand for the multiset (list) with elements $d_{1}, \ldots, d_{k}$ such that the element $d_{i}$ occurs in $L$ exactly $a_{i}$ times.

A list (multiset) $L$ will be called linearly realizable if there exists a path $P$ on the set of vertices $[1,|L|+1], P=\left(v_{1}, \ldots, v_{|L|+1}\right)$ such that

$L=\left\{\left|v_{i+1}-v_{i}\right|: i=1, \ldots,|L|\right\}$.

To emphasize the distinction between linearly realizable and realizable lists, we will sometimes call the (previously defined) realizable lists cyclically realizable.

Clearly, if $P$ is a linear realization of a list $L=\left\{d_{1}{ }^{a_{1}}, \ldots, d_{k}{ }^{a_{k}}\right\}$ with $\max \left\{d_{1}, \ldots, d_{k}\right\} \leq$ $\frac{|L|}{2}$, then $P$ is a cyclic realization of $L$ as well.

Main results of this section are the following four statements.

Theorem 3.1 Let $p=2 n+1, L=\left\{d_{0}{ }^{a_{0}}, d_{1}{ }^{a_{1}}, \ldots, d_{k}{ }^{a_{k}}\right\}, n \geq d_{1}>d_{2}>\ldots>d_{k}$, and $a_{i} \leq 2$, for $i=1, \ldots, k$. If $a_{0} \geq d_{1}-k+t-r$, where $t=\max \left\{d_{i} ; i>0, a_{i}=2\right\}$ and $r=\left|\left\{d_{i} ; i>0, a_{i}=2\right\}\right|$, then $L$ is (cyclically) realizable.

Theorem 3.2 Let $p=2 n+1$, and let $L=\left\{d^{a}, t^{b}\right\}$, where $d \leq n, t \leq n$, and $a+b=2 n$. Then $L$ is (cyclically) realizable.

Corollary 3.3 Let $L=\left\{1^{a_{1}}, 2^{a_{2}}, \ldots, n^{a_{n}}\right\}$, and suppose there is $j \in\{1,2, \ldots, n\}$ such that

$$
\sum_{1 \leq i \leq n, i \neq j} a_{i} \leq 4
$$

Then $L$ has a realization.

Theorem 3.4 Let $L=\left\{d_{1}^{a_{1}}, \ldots, d_{k}{ }^{a_{k}}\right\}$. Then there exists an $s_{0}$ so that for all $s \geq s_{0}$ the multiset $L^{\prime}=L \cup\left\{1^{s}\right\}$ is both, linearly and cyclically realizable.

Remark. For the sake of generality we will assume in the proof of Theorem 3.4 that $d_{i} \neq 1, i=1, \ldots, k$. However, it is easy to see that the proof will hold also in the case when $d_{i}=1$ for some $i, 1 \leq i \leq k$. Theorem 3.4 could be reformulated as follows.

Theorem 3.5 Let $L=\left\{d_{1}{ }^{a_{1}}, d_{2}{ }^{a_{2}}, \ldots, d_{k}{ }^{a_{k}}\right\}$. Then there exists an $s_{0}$ such that for all $s \geq s_{0}$, the list $L^{\prime}=\left\{d_{1}^{s}, d_{2}{ }^{a_{2}}, \ldots, d_{k}{ }^{a_{k}}\right\}$ is cyclically realizable whenever $1+s+\sum_{i=2}^{k} a_{i}$ is relatively prime to $d_{1}$.

For the sake of brevity, a path from a vertex $u$ to a vertex $v$ will be called a $u-v$ path.

Definition A set of vertex disjoint paths $P_{i} i=1, \ldots, s$, where $P_{i}$ is a $(v+i)-(w+i)$ path will be called a set of consecutive paths from $[v+1, v+s]$ to $[w+1, w+s]$.

Lemma 3.6 Let $\mathcal{C}$ be a set of $s$ consecutive paths from $[v+1, v+s]$ to $[w+1, w+s]$. Then there is a path $P$ so that $V(P)=\bigcup_{T \in \mathcal{C}} V(T), E(P) \supset \bigcup_{T \in \mathcal{C}} E(T), d(P)=\left\{1^{s-1}\right\} \cup$ $\bigcup_{T \in \mathcal{C}} d(T)$ so that, for $s$ odd, 
(i) $P$ is $a(v+1)-(w+s)$ path;

(ii) $P$ is $a(w+1)-(w+s)$ path and $\{v+1, v+2\}$ is an edge of $P$.

Proof. To obtain the desired path add to the edges of paths $T^{\prime}$ the following edges of length 1:

(i) $\{v+2, v+3\},\{v+4, v+5\}, \ldots,\{v+s-1, v+s\}$ and $\{w+1, w+2\},\{w+3, w+$ $4\}, \ldots,\{w+s-2, w+s-1\}$;

(ii) $\{v+1, v+2\},\{v+3, v+4\}, \ldots,\{v+s-1, v+s\}$ and $\{w+2, w+3\},\{w+4, w+$ $5\}, \ldots,\{w+s-2, w+s-1\}$.

In Fig. 1 and Fig. 2 the previous lemmas are illustrated for $s$ even and $s$ odd, respectively.

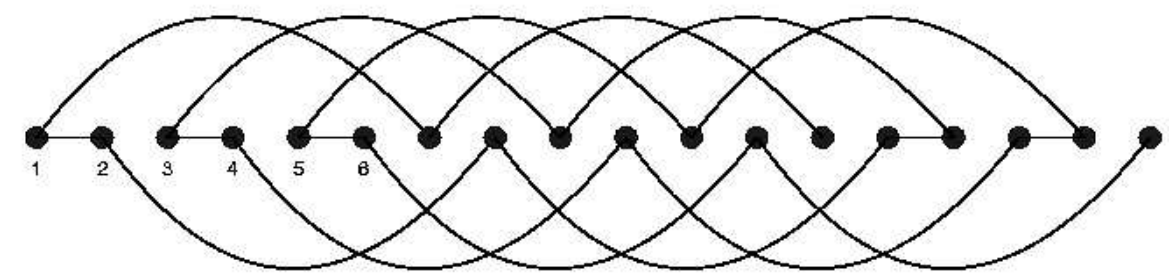

Figure 1

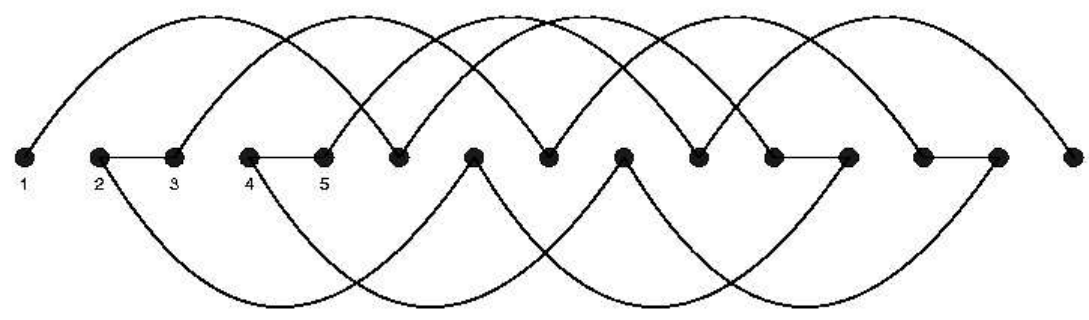

Figure 2

Lemma 3.7 Let $L=\left\{d_{1}^{a_{1}}, \ldots, d_{k}^{a_{k}}\right\}$, where $1<d_{1}<d_{2}<\cdots<d_{k}$, and $d_{i} \mid a_{i}$, for $i=1, \ldots, k$. Then for $L^{\prime}=L \cup\left\{1^{d_{k}-1}\right\}$ there exists: (i) for $d_{1}, \ldots, d_{k}$ being odd, an $1-\left(\left|L^{\prime}\right|+1\right)$ path $P$ which is a linear realization of $L^{\prime}$; (ii) for $d_{1}, \ldots, d_{k}$ being even, a $\left(\left|L^{\prime}\right|-d_{k}+2\right)-\left(\left|L^{\prime}\right|+1\right)$ path $P$ which is a linear realization of $L^{\prime}$, and $\{1,2\}$ is an edge of $P$.

Proof. A path $P$ with the required properties will be constructed successively. By the $\left(d^{a}, v\right)$ path we will understand the path $T=(v, v+d, v+2 d, \ldots, v+a d)$. Obviously, $d(T)=\left\{d^{a}\right\}$. Further, set $t_{i}=\frac{a_{i}}{d_{i}}$. We start with $d_{1}$ consecutive paths $P_{1}, \ldots, P_{d_{1}}$, where $P_{i}$ is the $\left(d_{1}^{t_{1}}, i\right)$ path from $\left[1, d_{1}\right]$ to $\left[a_{1}+1, a_{1}+d_{1}\right]$. Clearly, $\bigcup_{i=1}^{d_{1}} d\left(P_{i}\right)=\left\{d_{1}{ }^{a_{1}}\right\}$ and 
$\bigcup_{i=1}^{d_{1}} V\left(P_{i}\right)=\left[1, a_{1}+d_{1}\right]$. Now consider $d_{2}$ consecutive paths $P_{1}{ }^{\prime}, \ldots, P_{d_{2}}{ }^{\prime}$, where $P_{i}{ }^{\prime}$ is the $\left(d_{2}^{t_{2}}, a_{1}+i\right)$ path. It is easy to check that $P_{i}^{\prime} s$ are consecutive paths from $\left[a_{1}+1, a_{1}+d_{2}\right]$ to $\left[a_{1}+a_{2}+1, a_{1}+a_{2}+d_{2}\right]$ with $\bigcup_{i=1}^{d_{2}} d\left(P_{i}^{\prime}\right)=\left\{d_{2}{ }^{a_{2}}\right\}$, and $\bigcup_{i=1}^{d_{2}} V\left(P_{i}^{\prime}\right)=\left[a_{1}+1, a_{1}+a_{2}+d_{2}\right]$. Note that, for $i=1, \ldots, d_{1}$, the initial vertex of $P_{i}^{\prime}$ coincides with the terminal vertex of $P_{i}$. So at this stage we have two collections $\mathcal{C}_{1}, \mathcal{C}_{2}$ of consecutive paths. The collection $\mathcal{C}_{1}$ contains $d_{1}$ paths from $\left[1, d_{1}\right]$ to $\left[a_{1}+a_{2}+1, a_{1}+a_{2}+d_{1}\right]$, while $\mathcal{C}_{2}$ is a set of $d_{2}-d_{1}$ consecutive paths from $\left[a_{1}+d_{1}+1, a_{1}+d_{2}\right]$ to $\left[a_{1}+a_{2}+d_{1}+1, a_{1}+a_{2}+d_{2}\right]$. Formally, $\mathcal{C}_{1}=\left\{P_{1} \cup P_{1}^{\prime}, P_{2} \cup\right.$ $\left.P_{2}^{\prime}, \ldots, P_{d_{1}} \cup P_{d_{1}}{ }^{\prime}\right\}$, and $\mathcal{C}_{2}=\left\{P_{d_{1}+1}{ }^{\prime}, P_{d_{1}+2}{ }^{\prime}, \ldots, P_{d_{2}}{ }^{\prime}\right\}$. Thus, in aggregate we have $d_{2}$ vertex disjoint paths $T_{i}$ in $\mathcal{C}_{1} \cup \mathcal{C}_{2}$ with $\bigcup_{i=1}^{d_{2}} d\left(T_{i}\right)=\left\{d_{1}{ }^{a_{1}}, d_{2}{ }^{a_{2}}\right\}$, and $\bigcup_{i=1}^{d_{2}} V\left(T_{i}\right)=\left[1, a_{1}+a_{2}+d_{2}\right]$. In the same way as above we add now $d_{3}$ consecutive paths $P_{i}^{\prime \prime}, i=1, \ldots, d_{3}$, where $P_{i}^{\prime \prime}$ is a $\left(d_{3}{ }^{t_{3}}, i+a_{1}+a_{2}\right)$ path. Thus, $P_{i}^{\prime \prime}$ s are consecutive paths from $\left[a_{1}+a_{2}+1, a_{1}+a_{2}+d_{3}\right]$ to $\left[a_{1}+a_{2}+a_{3}+1, a_{1}+a_{2}+a_{3}+d_{3}\right]$ with $\bigcup_{i=1}^{d_{3}} d\left(P_{i}^{\prime \prime}\right)=\left\{d_{3}{ }^{a_{3}}\right\}$, and $\bigcup_{i=1}^{d_{3}} V\left(P_{i}^{\prime \prime}\right)=$ $\left[a_{1}+a_{2}+1, a_{1}+a_{2}+a_{3}+d_{3}\right]$. For $i=1, \ldots, d_{1}$, the initial vertex of $P_{i}^{\prime \prime}$ coincides with the terminal vertex of a path in the collection $\mathcal{C}_{1}$, and for $i=d_{1}+1, \ldots, d_{2}$, with the terminal vertex of a path in the collection $\mathcal{C}_{2}$. At this stage we have three collections $\mathcal{C}_{1}{ }^{\prime}, \mathcal{C}_{2}{ }^{\prime}$, and $\mathcal{C}_{3}{ }^{\prime}$ of consecutive paths $T_{i}$ in aggregate. The paths in $\mathcal{C}_{1}{ }^{\prime}$ and $\mathcal{C}_{2}{ }^{\prime}$ have been obtained by an extension from paths in $\mathcal{C}_{1}$ and $\mathcal{C}_{2}$, respectively. So they have the same initial vertex as before, their terminal vertices are now in $\left[a_{1}+a_{2}+a_{3}+1, a_{1}+a_{2}+a_{3}+d_{1}\right]$, and in $\left[a_{1}+a_{2}+a_{3}+d_{1}+1, a_{1}+a_{2}+a_{3}+d_{2}\right]$, respectively. Further, there are $d_{3}-d_{2}$ consecutive paths in $\mathcal{C}_{3}{ }^{\prime}$ from $\left[a_{1}+a_{2}+d_{2}+1, a_{1}+a_{2}+d_{3}\right]$ to $\left[a_{1}+a_{2}+a_{3}+d_{2}+1, a_{1}+a_{2}+a_{3}+d_{3}\right]$. It is easy to see that $\bigcup_{i=1}^{d_{3}} d\left(T_{i}\right)=\left\{d_{1}{ }^{a_{1}}, d_{2}{ }^{a_{2}}, d_{3}{ }^{a_{3}}\right\}$, and $\bigcup_{i=1}^{d_{3}} V\left(T_{i}\right)=\left[1, a_{1}+a_{2}+a_{3}+d_{3}\right]$. Thus, $\mathcal{C}_{1}^{\prime}=\left\{P_{1} \cup P_{1}^{\prime} \cup P_{1}^{\prime \prime}, P_{2} \cup P_{2}{ }^{\prime} \cup P_{2}^{\prime \prime}, \ldots, P_{d_{1}} \cup P_{d_{1}}{ }^{\prime} \cup P_{d_{1}}{ }^{\prime \prime}\right\}, \mathcal{C}_{2}{ }^{\prime}=\left\{P_{d_{1}+1}{ }^{\prime} \cup P_{d_{1}+1}{ }^{\prime \prime}, P_{d_{1}+2}{ }^{\prime} \cup\right.$ $\left.P_{d_{1}+2}{ }^{\prime \prime}, \ldots, P_{d_{2}}{ }^{\prime} \cup P_{d_{2}}{ }^{\prime \prime}\right\}$, and $\mathcal{C}_{3}{ }^{\prime}=\left\{P_{d_{2}+1}{ }^{\prime \prime}, P_{d_{2}+2}{ }^{\prime \prime}, \ldots, P_{d_{3}}{ }^{\prime \prime}\right\}$.

By repeatedly applying the above procedure, we will construct $d_{k}$ vertex-disjoint paths $T_{i}, i=1, \ldots, d_{k}$ so that $\bigcup_{i=1}^{d_{k}} d\left(T_{i}\right)=L$, and $\bigcup_{i=1}^{d_{k}} V\left(T_{i}\right)=\left[1, a_{1}+a_{2}+\ldots+a_{k}+d_{k}\right]=$ $\left[1,|L|+d_{k}\right]=\left[1,\left|L^{\prime}\right|+1\right]$ that can be partitioned into $k$ collections of consecutive paths $\mathcal{C}_{1}{ }^{*}, \mathcal{C}_{2}{ }^{*}, \ldots, \mathcal{C}_{k}{ }^{*}$. There are $d_{1}, d_{2}-d_{1}, \ldots, d_{k}-d_{k-1}$ consecutive paths in these collections, respectively. For each $i$, the paths in $\mathcal{C}_{i}{ }^{*}$ are consecutive paths from $\left[a_{1}+\cdots+a_{i-1}+\right.$ $\left.d_{i-1}+1, a_{1}+\cdots+a_{i-1}+d_{i}\right]$ to $\left[a_{1}+\ldots+a_{k}+d_{i-1}+1, a_{1}+\ldots+a_{k}+d_{i}\right]$; here we have set for convenience $d_{0}=a_{0}=0$. We can describe the given collections of paths formally as follows: Set $\alpha_{0}=0$ and $\alpha_{i}=\sum_{j=1}^{k} \alpha_{j}$ for $i=1, .2, \ldots, k-1$. For each pair $(i, j)$ with $1 \leq i \leq k$ and $1 \leq j \leq d_{i}$, let $P_{i j}$ be the $\left(d_{i}{ }^{t_{i}}\right)-\left(\alpha_{i-1}+j\right)$ path. Then we have

$$
\begin{aligned}
& \mathcal{C}^{*}=\left\{\bigcup_{i=1}^{k} P_{i 1}, \bigcup_{i=1}^{k} P_{i 2}, \ldots, \bigcup_{i=1}^{k} P_{i, d_{1}}\right\} ; \\
& \mathcal{C}_{2}{ }^{*}=\left\{\bigcup_{i=2}^{k} P_{i, d_{1}+1}, \bigcup_{i=2}^{k} P_{i, d_{1}+2}, \ldots, \bigcup_{i=2}^{k} P_{i, d_{2}}\right\} ; \\
& \ldots
\end{aligned}
$$




$$
\begin{aligned}
& \mathcal{C}_{h}{ }^{*}=\left\{\bigcup_{i=h}^{k} P_{i, d_{h-1}+1}, \bigcup_{i=h}^{k} P_{i, d_{h-1}+2}, \ldots, \bigcup_{i=h}^{k} P_{i, d_{h}}\right\} ; \\
& \ldots \\
& \mathcal{C}_{k}^{*}=\left\{P_{k, d_{k-1}+1}, P_{k, d_{k-1}+2}, \ldots, P_{k, d_{k}}\right\} .
\end{aligned}
$$

Suppose that $d_{1}, \ldots, d_{k}$ are even. Applying Lemma 3.6 (ii) to collections $\mathcal{C}_{1}{ }^{*}, \ldots, \mathcal{C}_{k}{ }^{*}$ we obtain $k$ paths $S_{1}, \ldots, S_{k}$ so that the initial vertex of $S_{1}$ is the vertex $a_{1}+\cdots+a_{k}+1$, the initial vertex of $S_{i}, i=2, \ldots, k$ is the vertex consecutive to the terminal vertex of $S_{i-1}$, and the terminal vertex of $S_{k}$ is the vertex $a_{1}+\cdots+a_{k}+d_{k}=\left|L^{\prime}\right|+1$. Adding $k-1$ suitable edges of length 1 results in the sought path $P$.

Let now $d_{1}, \ldots, d_{k}$ be odd. Applying Lemma 3.6(i) to the collection of paths in $\mathcal{C}_{1}{ }^{*}$, and Lemma 3.6(ii) to the collections $\mathcal{C}_{2}{ }^{*}, \ldots, \mathcal{C}_{k}{ }^{*}$ we obtain $k$ paths $S_{1}, \ldots, S_{k}$ so that the initial vertex of $S_{1}$ is the vertex 1 , the initial vertex of $S_{i}, i=2, \ldots, k$, is the vertex consecutive to the terminal vertex of $S_{i-1}$, and the terminal vertex of $S_{k}$ is the vertex $a_{1}+\cdots+a_{k}+d_{k}=\left|L^{\prime}\right|+1$. Adding $k-1$ edges of length 1 results in a sought path $P$.

The construction used in the above proof can be utilized to extend the result of Lemma 3.7 to a more general case.

Lemma 3.8 Let $L=\left\{1^{d-1}, d^{a}\right\}, a=q d+r, 0 \leq r<d$, where $a \geq d$, and for $d$ odd, $r$ is even. Then there is a path $P$ with terminal vertex $|L|+1$ which is a linear realization of $L$.

Proof. Let $a=q d+r, 0 \leq r<d$. Construct $d$ paths $P_{i}, i=1, \ldots, d$, so that, for $i=1, \ldots, r$, the path $P_{i}$ is the $\left(d^{q+1}, i\right)$ path, and for $i=r+1, \ldots, d$ the path $P_{i}$ is the $\left(d^{q}, i\right)$ path. For $r=0$ it suffices to apply Lemma 3.7 with $k=1$. Otherwise, $\mathcal{C}^{\prime}=\left\{P_{i} ; i=1, \ldots, r\right\}$ is a set of consecutive paths from $[1, r]$ to $[a+d-r+1, a+d]$, and $\mathcal{C}^{\prime \prime}=\left\{P_{i} ; i=r+1, \ldots, d\right\}$ is a set of consecutive paths from $[r+1, d]$ to $[a+1, a+d-r]$.

Now we are going to consider three cases. For both $d$ and $a$ even, $\mathcal{C}^{\prime}$ and $\mathcal{C}^{\prime \prime}$ have an even number of paths. Applying Lemma 3.6(ii) to both $\mathcal{C}^{\prime}$ and $\mathcal{C}^{\prime \prime}$ we obtain an $(a+d-r+1)-(a+d)$ path $P^{\prime}$ which contains the edge $\{1,2\}$ and a $(a+1)-(a+d-r)$ path $P^{\prime \prime}$. Clearly the path $P$ with $E(P)=E\left(P^{\prime}\right) \cup E\left(P^{\prime \prime}\right) \cup\{a+d-r, a+d-r+1\}$ has the required properties. For $a$ odd, to construct the path $P$ it suffices to construct the path $P$ for $a-1$ and then to extend it with the edge $\{a, a+d\}$. For $d$ odd and $r$ even $C^{\prime}$ contains an even number of paths while $\mathcal{C}^{\prime \prime}$ contains an odd number of paths. Applying Lemma 3.6(ii) to $\mathcal{C}^{\prime}$ and Lemma 3.6(i) to $\mathcal{C}^{\prime \prime}$ we obtain a $\left.(a+d-r+1)-(a+d)\right)$ path $P^{\prime}$ and $(r+1)-(a+d-r)$ path $P^{\prime \prime}$. Thus the initial vertex of $P^{\prime}$ is a vertex consecutive to the terminal vertex of $P^{\prime \prime}$ and the terminal vertex of $P^{\prime}$ is the last vertex $a+d$. Adding the edge $(u, v)$ of length 1 results in a desired path.

Lemma 3.9 Let $L=\left\{d_{1}^{a_{1}}, \ldots, d_{k}^{a_{k}}\right\}$, where $a_{i}$, $d_{i}$ are even, $1<d_{1}<d_{2}<\cdots<d_{k}$, and $d_{i} \leq a_{i}$, for $i=1, \ldots, k$. Set $L^{\prime}=L \cup\left\{1^{d_{k}-1}\right\}$. Then there exists a $\left(\left|L^{\prime}\right|-d_{k}\right)-\left(\left|L^{\prime}\right|+1\right)$ path $P$ which is a linear realization of $L^{\prime}$, and $\{1,2\}$ is an edge of $P$.

Proof. For $i=1, \ldots, k$ let $a_{i}=q_{i} d_{i}+r_{i}$, where $0 \leq r_{i}<d_{i}$. First we construct $d_{1}$ paths $P_{i}, i=1, \ldots, d_{1}$, each of them a $\left(d_{1}^{x}, i\right)$ path where $x=q_{1}+1$ for $i=1, \ldots, r_{1}$, and $x=q_{1}$ otherwise. As in the proof of Lemma 3.7, the paths $P_{i}$ form either one, for 
$r_{1}=0$, or two sets of consecutive paths of even cardinality, for $r_{1}>0$. Now we construct $d_{2}$ paths $P_{i}^{\prime}$, so that $P_{i}^{\prime}$ is a $\left(d_{2}{ }^{x}, a_{1}+i\right)$ path, where $x=q_{2}+1$ for $i=1, \ldots, r_{2}$, and $x=q_{2}$ otherwise. Clearly, for $i=1, \ldots, d_{1}$, the initial vertex of the path $P_{i}^{\prime}$ coincides with the terminal vertex of a path $P_{j}$ for some $j \in\left[1, d_{1}\right]$. At this moment we have $d_{2}$ vertex disjoint paths $T_{i}$ so that $\bigcup_{i=1}^{d_{2}} V\left(T_{i}\right)=\left[1, a_{1}+a_{2}+d_{2}\right]$, and $\bigcup_{i=1}^{d_{2}} d\left(T_{i}\right)=\left\{d_{1}{ }^{a_{1}}, d_{2}{ }^{a_{2}}\right\}$. Further,with respect to $r_{1}$ and $r_{2}$, the paths $T_{i}$ form either 2, or 3 , or 4 sets of consecutive paths, so that each set contains an even number of paths. We consider $d_{3}$ paths $P_{i}^{\prime \prime}$, each of them a $\left(d_{3}^{x}, a_{1}+a_{2}+i\right)$ path where $x=q_{3}+1$ for $i=1, \ldots, r_{3}$, and $x=q_{3}$ otherwise. The initial vertex of the first $d_{2}$ paths $P_{i}^{\prime \prime}$ coincides with the terminal vertex of a path $T_{i}$. So at this moment we have $d_{3}$ paths $T_{i}^{\prime}$ that form, with respect to the values of $r_{1}, r_{2}$, and $r_{3}$ between 3 and 6 sets of consecutive paths, each having an even number of elements. By repeatedly applying the above procedure we will obtain a set of $d_{k}$ paths $S_{i}$ so that $\bigcup_{i=1}^{d_{k}} V\left(S_{i}\right)=\left[1, a_{1}+\cdots+a_{k}+d_{k}\right]$, and $\bigcup_{i=1}^{d_{k}} d\left(T_{i}\right)=\left\{d_{1}{ }^{a_{1}}, d_{2}{ }^{a_{2}}, \ldots, d_{k}{ }^{a_{k}}\right\}$. These $d_{k}$ paths can be partitioned into $m, k \leq m \leq 2 k$, sets $\mathcal{C}_{i}$ of consecutive paths, each having an even number of paths. The union of the terminal vertices of paths in $\mathcal{C}_{i}$ s is the interval $\left[a_{1}+\ldots+a_{k}, a_{1}+\ldots+a_{k}+d_{k}\right]$. Applying Lemma 3.6(ii) to each set $\mathcal{C}_{i}$ of paths results in obtaining paths $T_{i}, i=1, \ldots, m$, so that the terminal vertex of $T_{i}$ is followed by the initial vertex of the path $T_{i+1}$, for $i=1, \ldots, m-1$, and the terminal vertex of $T_{m}$ is the last vertex $\left|L^{\prime}\right|+1$. Adding the needed edges of length 1 leads to the required path $T$. As the total number of paths in the sets $\mathcal{C}_{i} \mathrm{~s}$ is $d_{k}$, we used in aggregate $d_{k}-1$ edges of length 1 to construct the path $T$.

Lemma 3.10 Let $L=\left\{1^{d-1}, d^{a}\right\}$, where $a \leq d$. (i) For a odd, there is a $(|L|)-(|L|+1)$ path $P$ which is a linear realization of $L$, and $\{1,2\}$ is an edge of $P$; (ii) for a even, there is an $(|L|-d+2)-(|L|+1)$ path $P$ which is a linear realization of $L$, and $\{1,2\}$ is an edge of $P$. That is, the terminal vertex of $P$ is the last vertex of $V(P)$.

Proof. Let $a$ be odd. Take $a$ edges $\{i, i+d\}, i=1, \ldots, a$ of length $d$. By adding $a-2$ edges $\{1,2\},\{3,4\},\{5,6\}, \ldots,\{a-2, a-1\},\{d+2, d+3\},\{d+4, d+5\}, \ldots,\{d+a-3, d+$ $a-2\}$ and the edges of the path $S=(a, a+1, a+2, \ldots, d-1, d, d+1)$ we get the desired path $P$. Now, let $a$ be even. Remove from the path $P$ constructed for $L=\left\{1^{d-1}, d^{a+1}\right\}$ the edge $\{a+1, a+d+1\}$. The resulting path has the required properties.

Lemma 3.11 For $i=1,2$, let $L_{i}$ be a multiset and each $P_{i}$ be $a\left(\left|L_{i}\right|\right)-\left(\left|L_{i}\right|+1\right)$ path, which is a linear realization of $L_{i}$, and let $\{1,2\}$ be an edge of $P_{i}$. Set $L=L_{1} \cup L_{2}-\left\{1^{1}\right\}$. Then there is a $(|L|)-(|L|+1)$ path $P$ which is a linear realization of $L$, and $\{1,2\}$ is an edge of $P$.

Proof. To obtain the desired path remove the edge $\{1,2\}$ from $P_{2}$ and shift the other edges of $P_{2}$ to the right by $\left|L_{1}\right|$. Note that the original vertices 1,2 of $P_{2}$ will be identified with the endvertices of $P_{1}$.

Lemma 3.12 Let $L=\left\{d_{1}^{a_{1}}, \ldots, d_{k}{ }^{a_{k}}\right\}$, where $d_{i}>1, a_{i}$ are odd, and $a_{i} \leq d_{i}$ for $i=$ $1, \ldots, k$. Then there is a $\left(\left|L^{\prime}\right|\right)-\left(\left|L^{\prime}\right|+1\right)$ path $P$ that is a linear realization of $L^{\prime}=L \cup$ $\left\{1^{s}\right\}, s \geq d_{1}+d_{2}+\cdots+d_{k}-2 k+1$, and $\{1,2\}$ is an edge of $P$. 
Proof. We obtain the desired path by repeatedly using Lemma 3.11 and Lemma 3.10(i).

Lemma 3.13 Let $L=\left\{1^{s}, d_{1}^{a_{1}}, \ldots, d_{k}{ }^{a_{k}}\right\}$, where $s=d_{1}-k, d_{1}>d_{2}>\cdots>d_{k}>1$, and $a_{1}=\cdots=a_{k}=1$. Then there is a path $P$ which is a linear realization of $L$ so that 1 is the initial vertex of $P$.

Proof. Let us define, iteratively, $k$ paths $P_{1}, \ldots, P_{k}$ according to the following rules. For convenience we set $d_{k+1}=0$. Set $P_{0}=(1)$, i.e., $P_{0}$ is a path of length 0 . Suppose that $P_{i-1}, i-1 \geq 0$, has been already constructed. Then $P_{i}$ is obtained from $P_{i-1}$ by extending $P_{i-1}$ first by an edge of length $d_{i}$ followed by $d_{i}-d_{i+1}-1$ edges of length 1 . More precisely, let $v$ be the terminal vertex of $P_{i-1}$. Then, for $i$ odd, we extend $P_{i-1}$ by the edge $v, v+d_{i}$, followed by $d_{i}-d_{i+1}-1$ edges $\left\{v+d_{i}-j, v+d_{i}-j-1\right\}$ of length 1 , for $j=0,1, \ldots, d_{i}-d_{i+1}-2$; for $i$ even, by the edge $\left\{v, v-d_{i}\right\}$, followed by $d_{i}-d_{i+1}-1$ edges $\left\{v-d_{i}+j, v+d_{i}+j+1\right\}$ of length 1 , for $j=0,1, \ldots, d_{i}-d_{i+1}-2$. Then the required path $P$ is $P=P_{k}$

Proof of Theorem 3.1 First of all, since $p=2 n+1$ is a prime, we may assume w.l.o.g. that $d_{0}=1$. By Lemma 3.13, there is a path $P_{1}$ that is a linear realization of $L_{1}=\left\{1^{d_{1}-k}, d_{1}{ }^{1}, \ldots, d_{k}{ }^{1}\right\}$, so that $\left|L_{1}\right|+1$ is the initial vertex of $P_{1}$. By the same lemma, there is a path $P_{2}$ that is a linear realization of $L_{2}=\left\{1^{s}, d_{1}{ }^{b_{1}}, \ldots, d_{k}{ }^{b_{k}}\right\}$, where $s=t-r$, $b_{i}=a_{i}-1$, and the vertex 1 is the initial vertex of $P_{2}$. Clearly, the total number of edges of length 1 in $P_{1}$ and $P_{2}$ is $m=d_{1}-k+t-r \geq 2 d_{1}-k$. To obtain a path $P$ that is a linear realization of $L$ it suffices to take the path $P_{1}$ followed by a path of length $a_{0}-m$ consisting of edges of length 1 which is in turn followed by path $P_{2}$. As $V(P)=[1,2 n+1]$ and the longest edge of $P$ has length at most $n$, the path $P$ is a (cyclic) realization of $L$ as well.

Fig. 3 illustrates the proof of the previous theorem for $L=\left\{1^{8}, 7^{1}, 4^{2}, 3^{1}, 2^{2}\right\}$ as well as the proof of Lemma 3.13 for $L_{1}=\left\{1^{3}, 7^{1}, 4^{1}, 3^{1}, 2^{1}\right\}$ and $L_{2}=\left\{1^{2}, 4^{1}, 2^{1}\right\}$.

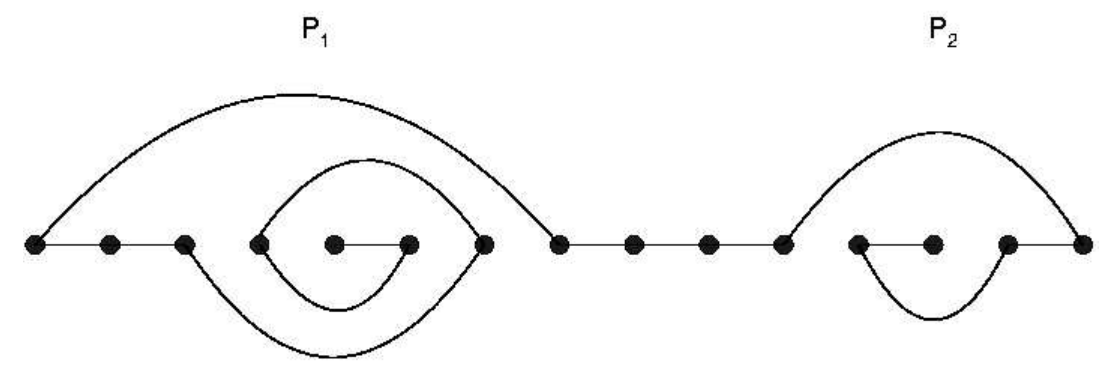

Figure 3

Proof of Theorem 3.2 W.l.o.g. we assume that $b \geq a$. As $p$ is a prime number, we may further assume that $t=1$. Let $a=p d+r, 0 \leq r<d$. First we consider the case where $a \leq d$ or $a>d$ and in case $d$ is odd then $r$ is even. Then, by Lemma 3.10 or by Lemma 3.8 there is a path $P$ that is a linear realization of $L^{\prime}=\left\{1^{d-1}, d^{a}\right\}$ so that the 
endvertex of $P$ is the last vertex of $\left[1,\left|L^{\prime}\right|+1\right]$. Adding $t-d+1$ edges of length 1 from the terminal vertex of $P$ results in a desired path.

So we are left with the case $a>d, d$ odd and $r$ odd. Construct $d$ paths as in the proof of Lemma 3.8. Then $\mathcal{C}^{\prime}=\left\{P_{i} ; i=1, \ldots, r\right\}$ is a set of consecutive paths from $[1, r]$ to $[a+d-r+1, a+d]$, and $\mathcal{C}^{\prime \prime}=\left\{P_{i} ; i=r+1, \ldots, d\right\}$ is a set of consecutive paths from $[r+1, d]$ to $[a+1, a+d-r]$. Applying Lemma 3.6(i), we get a $1-(a+d)$ path $P^{\prime}$. By adding the edges $\{a+d, a+d+1\},\{a+d+1, a+d+2\}, \ldots,\{q-2, q-1\},\{q-1, q\}$, and $\{1, q\}$ we obtain a cycle $C$ containing the path $P^{\prime}$. Further, by Lemma 3.6(ii), there is a path $P^{\prime \prime}$ with $a-d+r$ as its terminal vertex. To obtain the desired path $P$ we remove from $C$ the edge $\{a+d-r+1, a+d-r+2\}$ and finally add the edge $\{a+d-r, a+d-r+1\}$.

Remark. Note that in the above proof, as well as in the proof of Lemma 3.8, we used only the fact that $(t, p)=1$ but we have not used the fact that $(d, p)=1$ nor that $p$ is a prime.

Proof of Corollary 3.3 Since $p=2 n+1$ is a prime, we may assume w.l.o.g. that $j=1$. The statement then says that if $L$ is a partition of $2 n$ containing at most $s \leq 4$ non-ones then $L$ has a realization. This is obvious if $s=0$ or $s=1$; if $s=2$ then the statement follows either from Theorem 3.1, if there is $i>1$ so that $a_{i}=2$, or from Lemma 3.13 if there are $1<i<k$ so that $a_{i}=a_{k}=1$. For $s=3$, the statement follows either from Lemma 3.13, if there are $1<i<k<t$ so that $a_{i}=a_{k}=a_{t}=1$, or from Theorem 3.1, if there are $1<i, k$ so that $a_{1}+a_{k}=3$ or from Lemma 3.8, if there is $i>1, a_{i}=3$. When $s=4$, then the only case in which the statement does not follow from either Theorem 3.1, or Lemma 3.8, or Lemma 3.13, is when there are two indices $i, k \in\{2,3, \ldots, n\}$ such that $a_{i}=3$ and $a_{k}=1$. We consider this case next.

If $i=2$ (and $k \in\{3,4, \ldots, n\}$ ), i.e., there are three 2's in $L$, then e.g. the following hamiltonian path is a realization of $L$ :

$(0, k, k+1, k+2, \ldots, 2 n-1,2 n, 1,2,4,3,5,6,7, \ldots, k-2, k-1)$.

If $i=3$ and $k \in\{4,5, \ldots, n\}$ then e.g. the hamiltonian path

$(0,3, k+3, k+2, \ldots, 5,4,1,2,2 n, 2 n-1, \ldots, k+5, k+4)$,

while if $i=3$ and $k=2$ then e.g. the hamiltonian path

$(0,3,5,6, \ldots, 2 n-1,2 n, 2,1,4)$.

is a realization of $L$.

When $i \geq 4$ and $k \geq i+4$, then e.g. the hamiltonian path

$(k+3, k+4, \ldots, 2 n-1,2 n, 0, i, i-1, \ldots, 4,3, i+3, i+4, \ldots, k+1, k+2,2,1, i+1, i+2)$, when $i \geq 4$ and $3 \leq k \leq i+3$, then e.g. the hamiltonian path

$(i+2,1+1,1,2,2 n-k+3,2 n-k+4, \ldots, 2 n, 0,5,4,3, i+3, i+4, \ldots, 2 n-k+1,2 n-k+2)$, and finally, when $i \geq 4$ and $k=2$, then e.g. the hamiltonian path

$(i+2, i+1,1,0, i, i-1, \ldots, 4,2,3, i+3, i+4, \ldots, 2 n-1,2 n)$

is a realization of $L$.

This completes the proof of the Corollary.

We will deal now with the case when $L$ contains at least two distances greater than 1 . 
Proof of Theorem 3.4 By using lemmas proved above, a sought path $P$ can be constructed in several ways. Each of them will require a different number of edges of length 1 . The optimal way depends on the individual values of $d_{i}$, and $a_{i}$. The construction described here is only one of the possible ways to obtain $P$. First we partition $L$ into four parts $L_{1}, L_{2}, L_{3}, L_{4}$. Let $d^{a} \in L$. For $d$ even, if $a \geq d$ and $a$ even, then $d^{a} \in L_{2}$; for $a \geq d, a$ odd, we put $d^{a-1} \in L_{2}$ and $d^{1} \in L_{4}$; for $a<\bar{d}$ and $a$ odd, $d^{a} \in L_{1}$, for $a<d, a$ even, we put $d^{a-1} \in L_{1}$ and $d^{1} \in L_{4}$. Now we consider $d$ odd. For $a<d$ we have: if $a$ is odd, then $d^{a} \in L_{1}$, for $a$ even, we put $d^{a-1} \in L_{1}$ and $d^{1} \in L_{4}$. Finally, let $a \geq d$, and $a=q d+r$, where $0 \leq r<d$. For $r=0$ it is $d^{a} \in L_{3}$, for $r$ odd we have $d^{q d} \in L_{3}$ and $d^{r} \in L_{1}$, for $r>0, r$ even, it is $d^{q d} \in L_{3}$ and $d^{r-1} \in L_{1}$, and $d^{1} \in L_{4}$. In this way every $d^{a} \in L_{1}$ has $a<d$ and $a$ odd; every $d^{a} \in L_{2}$ has both $d$ and $a$ even and $a \geq d$; every $d^{a} \in L_{3}$ has $d$ a divisor of $a$; every $d^{a} \in L_{1}$ has $a=1$.

By Lemma 3.12 there is a $\left(\left|L^{\prime}\right|\right)-\left(\left|L^{\prime}\right|+1\right)$ path $P_{1}$ which is a linear realization of $L_{1}{ }^{\prime}=L_{1} \cup\left\{1^{s_{1}}\right\}$, the value of $s_{1}$ given in the lemma. Further, by Lemma 3.9, there is a path $P_{2}$ that is a linear realization of $L_{2}{ }^{\prime}=L_{2} \cup\left\{1^{s_{2}}\right\}$, the value of $s_{2}$ given in the lemma, so that $\{1,2\}$ is an edge of $P_{2}$, and $\left|L_{2}{ }^{\prime}\right|+1$ is a terminal vertex of $P_{2}$. Applying Lemma 3.11 we obtain a $\left(\left|L_{1}^{\prime}\right|+\left|L_{2}^{\prime}\right|\right)-\left(\left|L_{1}^{\prime}\right|+\left|L_{2}^{\prime}\right|+1\right)$ path $T$ which is a linear realization of $L_{1}^{\prime} \cup L_{2}^{\prime}-\{1\}$. Let $P_{3}$ be a $1-\left(\left|L_{3}{ }^{\prime}\right|+1\right)$ path which is a linear realization of $L_{3}{ }^{\prime}=L_{3} \cup\left\{1^{s_{3}}\right\}$, cf. Lemma 3.7, where the value of $s_{3}$ is given as well.

Let $P_{3}^{\prime}$ be the path obtainable by shifting $P_{3}$ to the right by $\left|L_{1}{ }^{\prime}\right|+\left|L_{2}{ }^{\prime}\right|$. Thus the endvertex of $T$ coincides with the initial vertex of $P_{3}{ }^{\prime}$. Then resulting path $T^{\prime}:=T \cup P_{3}{ }^{\prime}$ is a $\left(\left|L_{1}{ }^{\prime}\right|+\left|L_{2}{ }^{\prime}\right|\right)-\left(\left|L_{1}{ }^{\prime}\right|+\left|L_{2}{ }^{\prime}\right|+1\right)$ that is a linear realization of $L_{1}{ }^{\prime} \cup L_{2}{ }^{\prime} \cup L_{3}{ }^{\prime}-\{1\}$. Finally, let $L_{4}=\left\{t_{1}{ }^{1}, \ldots, t_{m}{ }^{1}\right\}, t_{1}>\cdots>t_{m}$, and let $P_{4}$ be a linear realization of $L_{4}{ }^{\prime} \cup\left\{1^{s_{4}}\right\}$, where $s_{4}=t_{1}-m$ so that 1 is the initial vertex of $P_{4}$, cf. Lemma 3.13.

Let $s \geq s_{0}:=s_{1}+s_{2}+s_{3}+s_{4}-1$ and let $P_{4}^{\prime}$ be the path obtainable by shifting $P_{4}$ to the right by $\left|L_{1}{ }^{\prime}\right|+\left|L_{2}{ }^{\prime}\right|+\left|L_{3}{ }^{\prime}\right|+s-s_{0}$. To obtain a path that is a linear realization of $L \cup\left\{1^{s}\right\}$ it suffices to insert $s-s_{0}$ edges of length 1 between the terminal vertex of $T^{\prime}$ and the initial vertex of $P_{4}^{\prime}$.

Identifying the terminal vertex of $T^{\prime}$ with the initial vertex of $P_{4}$ results in a path $P$ that is a linear realization of $L_{1}{ }^{\prime} \cup L_{2}{ }^{\prime} \cup L_{3}{ }^{\prime} \cup L_{4}{ }^{\prime}-\{1\}=L \cup\left\{1^{s_{0}}\right\}$, where $s_{0}=$ $s_{1}+s_{2}+s_{3}+s_{4}-1$. To obtain a path that is a linear realization of $L \cup\left\{1^{s}\right\}$ for $s \geq s_{0}$ it suffices to insert $s-s_{0}$ edges of length 1 in between the terminal vertex of $T^{\prime}$ and the initial vertex of $P_{4}^{\prime}$.

As mentioned above, a linear realization of $L$ is a cyclic realization of $L$ if and only if $d=\max \left\{d_{i}, i=1, \ldots, k\right\} \leq \frac{|L|}{2}$. If the condition is not satisfied, we only need to make $s_{0}$ sufficiently large.

Remark. To get an explicit bound on $s_{0}$ in terms of $d^{\prime} s$ and $a^{\prime} s$, one only needs to refer to lemmas used in the proof of the previous theorem.

\section{Two lengths in the general case}

In this section, the number of vertices is no longer assumed to be prime (nor an odd number, for that matter). In such a general setting, it appears that the problem is even 
more difficult than the original Buratti's conjecture.

The following theorem is an extension of Theorem 3.2.

Theorem 4.1 Let $q, d, t$ be natural numbers, and let $L=\left\{d^{a}, t^{b}\right\}$ be a multiset where $a+b=q-1, d, t \leq \frac{q}{2}$. Then $L$ is (cyclically) realizable if and only if $(q, d, t)=1$ and $(t, q)-1 \leq a \leq q-(d, q)$ (which is equivalent to $(d, q)-1 \leq b \leq q-(t, q))$.

Proof. We start with the necessity part of the statement. Let $P=\left(\begin{array}{llll}x_{0} & x_{1} & \ldots & x_{q-1}\end{array}\right)$ be a hamiltonian path which is a realization of $L$. Suppose at first that $(q, d, t)=r>1$. Then, for each vertex $x_{i}$ of $P$, we would have $x_{i}=x_{0}(\bmod r)$, which is a contradiction. Therefore $(d, t, q)=1$.

If $(d, q)=1$ then the upper bound on $a$ (and the lower bound on $b$ ) is trivial. Assume now that $(d, q)=r>1$ and $a>q-(d, q)=q-r$. Then $b \leq r-2$, that is, there are in $P$ at most $r-2$ edges of length $t$.

If the edges of length $t$ are removed from $P$, then $P$ splits into at most $r-1$ paths. As the total number of edges is $q-1$, at least one of these parts, say the part $T$, contains at least $\frac{q-1}{r-1}<\frac{q}{r}$ edges. However, all these edges are of length $d$ and therefore $T$ has to contain a cycle, a contradiction. So we have proved that $a \leq q-(d, q)$, which in turn implies, as $a+b=q-1$, that $(d, q)-1 \leq b$. This completes the proof of the necessity part.

The following obvious claim will provide the key ingredient to show that the condition is sufficient as well. As this claim is a part of graph theory folklore we omit its proof.

Claim. Let $G=P_{m} \times P_{k}$, that is, let $G$ be the Cartesian product of the paths with $m$ and $k$ vertices, respectively, and let the set $\{(i, j): 1 \leq i \leq m, 1 \leq j \leq k\}$ be the vertex set of $G$. Then there is a hamiltonian path in $G$ with $v$ vertical edges for every $v$, $m-1 \leq v \leq k \cdot m-k$.

Now we are ready to prove the sufficiency part. Assume first that $(d, t)=m>1$. Then, with regard to the automorphism $\phi: Z_{q} \rightarrow Z_{q}$, where $\phi(m)=1$, it suffices to prove that $L^{\prime}=\left\{\left(\frac{d}{m}{ }^{a}, \frac{t}{m}\right)^{b}\right\}$ is cyclically realizable. Therefore, from now on, we assume that $(d, t)=1$. Let $(d, q)=(t, q)=1$ as well, and let, w.l.o.g., $a \geq b$. With respect to the automorphism $\phi: Z_{q} \rightarrow Z_{q}$, where $\phi(d)=1$, we need to prove that $L^{\prime}=\left(1^{a}, \phi(t)^{b}\right)$ is cyclically realizable. However, this case is covered by Theorem 3.2.

So we are left with the case $(d, q)+(t, q)>2$. W.l.o.g. we may assume $(d, q)=r>1$. Let $R$ be the $r \times \frac{q}{r}$ rectangular grid with vertices $\left\{(i, j): 1 \leq i \leq r, 1 \leq j \leq \frac{q}{r}\right\}$. Assign to each vertex of the grid the label $l(i, j)=(i-1) t+(j-1) d(\bmod q)$. Clearly, $\{l(i, j)\}=Z_{q}$ as we have $(d, t)=1$. It is easy to see that the labels $l(i, j)$ of each row are elements of an orbit of the automorphism $\phi$ of $Z_{q}, \phi(d)=1$.

Now consider the graph $G=P_{r} \times P_{q}$, the Cartesian product of two paths on the grid $R$. Clearly, for each horizontal edge $\{u, v\}$ we have $\min |l(u)-l(v)|, q-|l(u)-l(v)|=d$, and for each vertical edge $\{u, v\}$ we have $\min |l(u)-l(v)|, q-|l(u)-l(v)|=t$. Therefore, to prove that the multiset $M=\left\{d^{a}, t^{b}\right\}$ is cyclically realizable it is sufficient to find a hamiltonian path in $G$ which contains exactly $b$ vertical edges (and thus necessarily $a$ horizontal edges). By the above Claim $G$ contains a hamiltonian path with $b$ vertical 
edges, that is, edges of length $t$, for each

$$
b \in\left[r-1, q-\frac{q}{r}\right]
$$

So all that is left to be shown is that there is a hamiltonian path in $G$ with $b \in$ $\left[q-\frac{q}{r}+1, q-(t, q)\right]$. Note, that $(d, t)=1$ implies $b \in\left[q-\frac{q}{r} \leq q-(d, q)\right]$. Consider two cases. First, let $(t, q)=1$. Then we must have $b \geq a$, as otherwise we would have $a+b>2\left(q-\frac{q}{r}+1\right)>q-1$, a contradiction.

The proof in this case follows from the remark after Theorem 3.2.

Finally, let $(t, q)=s>1$. Then by the above Claim there is a hamiltonian path with $a$ edges of length $d$ for each $a \in\left[s-1, q-\frac{q}{s}\right]$. As $a+b=q-1$, this in turn implies that there is a hamiltonian path in $G$ with $b$ edges of length $t$ for each

$$
b \in\left[q-1-\left(q-\frac{q}{s}\right), q-1-(s-1)\right]=\left[\frac{q}{s}-1, q-s\right]
$$

Comparing (1) and (2) completes the proof of the sufficiency part since $\frac{q}{s} \leq q-\frac{q}{r}$, i.e. $\frac{1}{s}+\frac{1}{r} \leq 1$ holds for all $s, r>1$.

\section{$5 \quad$ Final remarks}

Buratti's conjecture claims that if $p=2 n+1$ is a prime then every list $L=\left\{d_{1}{ }^{a_{1}}, d_{2}{ }^{a_{2}}, \ldots\right.$, $\left.d_{k}{ }^{a_{k}}\right\}$, where $\sum_{i=1}^{k} a_{i}=2 n$, is realizable. We believe that the property of $p$ being a prime is not necessary for the validity of Buratti's conjecture, and that it can be replaced by a weaker condition $\left(p, d_{i}\right)=1$ for $i=1, \ldots, k$. However, the weaker condition is still rather restrictive. One would like to know, given a general number $q$, which lists of cardinality $q-1$ are realizable. The treatment of the general case of two lengths in Section 4 and some additional experimental evidence suggests the following extension of Buratti's conjecture:

Conjecture. Let $L=\left\{d_{1}{ }^{a_{1}}, d_{2}{ }^{a_{2}}, \ldots, d_{k}{ }^{a_{k}}\right\},|M|=q-1$. Then $L$ is realizable if and only for each subset $J \subseteq[1, k], \sum_{j \in J} a_{j} \geq r-1$ where $r$ is the greatest common divisor of the numbers in the set $\{q\} \cup\left\{a_{i}: 1 \leq i \leq k, i \notin J\right\}$. That is, for each subset $J$ of the index set $[1, k]$, the sum of $a_{j}$ s must be at least as large as the g.c.d. $\left(A_{J}\right)-1$ where $A_{J}$ contains $q$ and all $a_{i}$ s that are not in $J$.

The necessity of the above conditions can be shown in the same way as the necessity of conditions in Theorem 4.1.

Note also that in the Conjecture, if $J=[1, k]$ then the above condition will read $\sum_{i=1}^{k} a_{i} \geq q-1$.

It is not difficult to see that for $k=2$ the above conditions reduce to the conditions of Theorem 1.

The Conjecture has been verified for all $n \leq 18$ [3]. As for the original Buratti's conjecture, which has been verified for all primes $p \leq 23$, numerical evidence gathered by Meszka [3] for all realizations of all lists in the case of primes $p \leq 13$ suggests that, roughly, the larger the number of non-zero frequencies of distances and the more uniform their distribution, the larger the number of corresponding realizations. The minimum number 
of realizations is attained for the list containing only one distance, while apparently the list with the largest number of realizations is the list where each possible distance occurs exactly twice.

\section{References}

[1] M. Buratti: Private communication.

[2] S. Even: Graph Algorithms. Computer Science Press, 1979

[3] M. MeszKa: Private communication. 